\title{
Development of the MARPEX App Embedding the Mobile Augmented Reality Factors for Learning Motivation in Science Experiments
}

\author{
https://doi.org/10.3991/ijim.v14i17.16641 \\ Valarmathie Gopalan ${ }^{(凶)}$, Juliana Aida Abu Bakar, Abdul Nasir Zulkifli \\ Universiti Utara Malaysia, Kedah, Malaysia \\ valar83gopalan@gmail.com
}

\begin{abstract}
Mobile Augmented Reality (MAR) has grown exponentially over the last four decades from imagination to real physical experience, especially in education. Previously, experts have completed the concepts of effectiveness, usability, acceptance and understanding in designing MAR applications to introduce and expose technological advances. However, most of these applications are addressed extensively for classroom science learning, while science experiments receive little attention. Students have to go through difficulties in understanding the phenomena of science and consequently become dissatisfied with their grasp on basic knowledge and eventually cannot pursue higher education or career related to science. This paper presents the development of Mobile Augmented Reality for Physics (MARPEX) application for high school students. The purpose of this application is to enhance the learning motivation in science experiments through the content of modern science. It aims to provide an individual learning experience for each student in science experiments. The MARPEX app design has gone through several phases of filtering and evaluation based on the specified objectives. This application needs to maintain the factors necessary to achieve this goal. This app has been designed and developed specifically for science (physics) experiments on the topic of electromagnetism. The application encrypts several experimental instances with the addition of good visualization to understand this phenomenon and has real-life experimental experience.
\end{abstract}

Keywords - Mobile Augmented Reality, Learning Motivation, Science Experiment, MARPEX.

\section{Introduction}

Lack of student enrollment in science is a global issue that needs immediate attention. Lack of experience significantly affects students' confidence to pursue a field. This is indicated through the enrollment of students in science or STEM based learning. The actual hands-on learning experience is important in science-related subject matters, as science is related to many phenomena that do not exist in the real world. Good visualization skills are required to understand and bring to life 
experiences within their respective environments. Thus, there is a growing interest among MAR experts to enhance the learning experience, engagement, effectiveness, acceptance of MAR use in science education. Content analysis identifies more than 20 previous studies between 2008-2019 on the application of MAR in science learning [1], [2], [3], [4], [5], [6], [7], [8], [9], [10], [11], [12], [13], [14], [15], [16],[17], [18], [19], [20], [21], [22], [49], and [50]. Though, there are studies indicated that AR in science is capable as a teaching tool which suits a classroom learning [23], [23], [24], [25], [26] and [27]. Among these studies, students' motivation problems are not realized. This is because students' motivation is an important concept in learning [51], [52]. After all, students' motivation increases their attention, confidence and satisfaction in science learning. Unfortunately, students have significant visualization constraints in science learning due to their inexperience [6] and [28]. Similarly, little is known about how to increase students' motivation in science experiments. Therefore, this paper discusses the design and development of the MARPEX app for students' motivation through MAR and also for validating the Mobile AR in science experiment on learning motivation (MARSELM) conceptual model.

\section{Technical Requirements}

The MARPEX app runs on Android smartphones or tablets with the addition of Graphical User Interfaces (GUI). GUI is a proven method for interacting with applications using items such as buttons, icons, and menus used by most modern operating systems (OS). The MARPEX app is developed with features such as navigation buttons, information buttons, and help buttons to enhance interactivity and the effects of experimental learning. The MARPEX app uses the smartphone screen to view the virtual objects.

Technical requirements are essential to meet the hardware to make the application fully functional and the user-friendly MARPEX app. The MARPEX app runs on the minimum operating system (OS) of Android 4.1 (Jelly Bean) and also includes a set of application programming interfaces (APIs). Next, the MARPEX app test conducted using the Samsung Note 5 Android device with Android OS.1 specification, 32GB of storage, $1.5 \mathrm{GHz}$ processor, 1440 x 2560-pixel resolution and 16-megapixel rear camera and works smoothly. The presence of a rear camera is essential in mobile devices for detecting AR markers.

These markers are usually rectangles of images that have been printed on a piece of paper and placed on the scene to identify the place where digital information is presented. Tracking methods involve registering what the camera captures and linking it to an image that a $3 \mathrm{D}$ computer produces. The interaction flow begins with the AR marker (on any stable surface), where the smartphone camera scans the image to reveal the encrypted material. The MARPEX app requires a solid surface image to act as a marker and is measured by the preferred font type, size and width and height. Then, scan the customized AR marker through the android device. As a result, the application displays 3D overlapping computer materials on the screen. The marker's sizes (width and height) were set during development. The size of the markers and the 
types of fonts used during customize the marker is essential, as the camera only displays encrypted $3 \mathrm{D}$ objects when recognizing the same size scale and target font type. For instance, if the target marker is twenty units in width and the camera moves from the left edge to the right edge of the marker, the image will remain at twenty units along the $\mathrm{x}$ and $\mathrm{y}$ axes [29] and [30]. The display layout describes the process involved in running MARPEX app including users, android devices and target markers. To start the app, users must start the application from the smartphone through a perspective view. To enable the application, users need to scan the printed AR markers. Once the marker, scanned and recognized by the device camera, 3D objects are tracked and displayed on the screen. Users can see a $360^{\circ}$ view of the added object as the user moves the smartphone camera within the marker area (as long as the device camera can detect the unique design of the AR maker). Further information on the use of AR markers will be discussed when discussing the development of the MARPEX app.

\section{MARPEX App Architecture}

This paper introduces the MARPEX app, where by the MARSELM conceptual model has been embedded in its development. The initial discussion on content making and initial design and development of the MARPEX app discusses our previous work [31]. This paper discusses in detail on technical requirements during the development of the MARPEX app. A two-dimensional (2D) architecture first designed for the MAR environment employed to develop the app. A 2D sketch on A4 paper intended to guide users in the development phases to illustrate how the interface and functions were executed. Thus, the 2D sketch is used in this study to design the MAR app architecture. The architecture consists of several components that include; content making (text, audio and animated 3D model), development tools, AR markers and scenes to complete the assembly procedure, as shown in Figure 1.

The architecture of the MARPEX app shows that the app design passes through different stages through the use of several tools. The utilization of the MARPEX app requires the interaction of the device's camera with the AR markers, as depicted in the development tools section. The MARPEX app architecture requires a new database created from the Vuforia, an AR online database [32], to set a target marker for each experiment. Single target images are selected with customized widths and dimensions for the MARPEX app. The image needs to be uploaded to be the target of the database. It enables the activation of the author section in the Unity 3D software [33]. Simulation objects are displayed on the mobile screen for users to interact with the MAR app. Java Software Development Kit (JDK) and Android Software Development Kit (SDK) are the software development tools to build the MARPEX app for Android phones and tablets. The JDK describes the objects or scenes used in the development phase and also store the enhanced content in the storage of the database. It also publishes the apk file for Android phones and tablets, which can be used to launch the MARPEX app. 


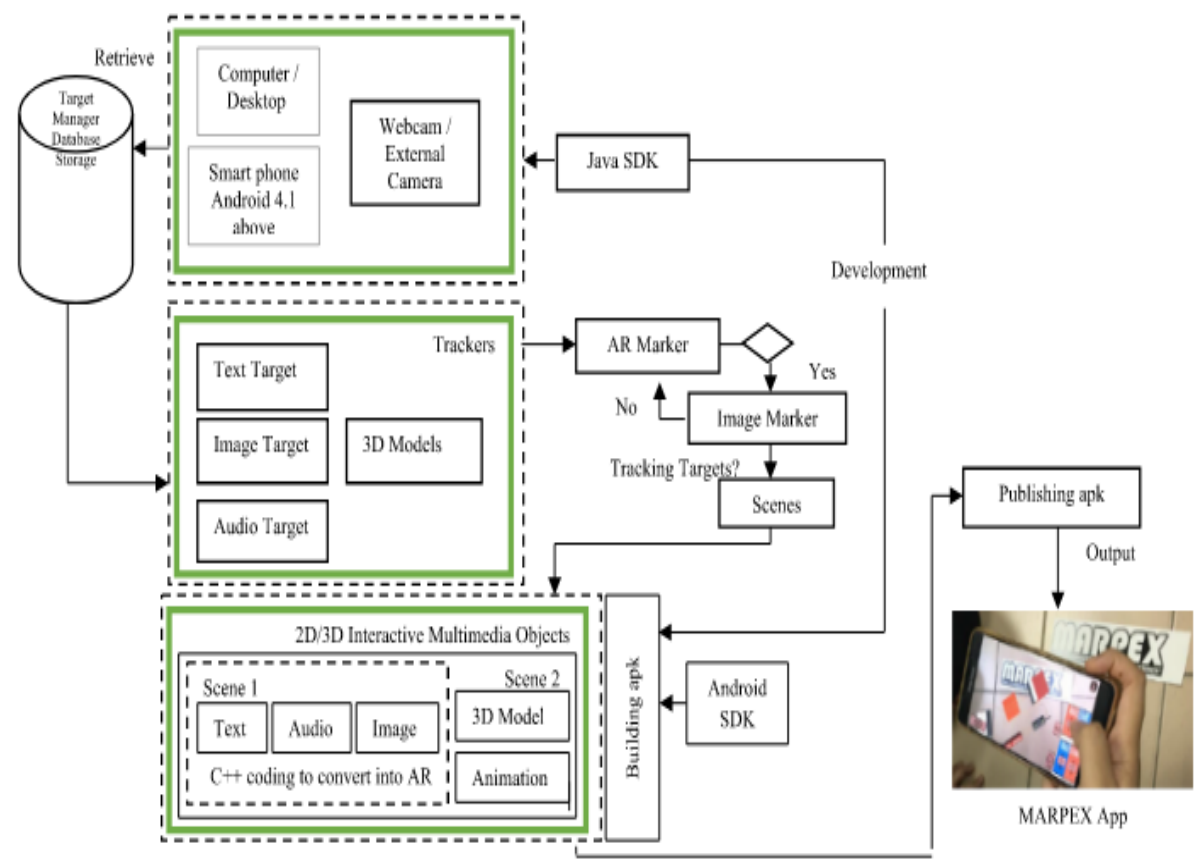

Fig. 1. Architecture of MARPEX App

\section{$4 \quad$ App Development}

The MARPEX app was designed specifically for enhancing students' motivation in a science experiment. The development phase involved with content making and integration of the MAR app on the android device. More than one software utilized in the development of the MARPEX app includes; science apparatus modeling (Blender), Android app development SDK (Vuforia SDK), and integration of MARPEX app into the android device (Unity3D version 2014).

\subsection{Contents of the MARPEX app}

The first stage in the development phase is content creation. It began with gathering information regarding the experiments and discussion with a content expert (science teachers).The content of the MARPEX app consists of an animated 3D model for the experiment section and text and audio effect for the quiz section. The key reason for allowing animated 3D models to be compatible with the three experiments is to suggest MAR in a science experiment. The ultimate purpose is to assist students' visualization of science phenomena and hands-on live experience doing experiments. Thus, it is vital to employ suitable media to convey the content to highlight visual senses due to the limitation in visualizing the science phenomenon. The contents of the MARPEX app tackle the experiment syllabus from the 
electromagnetism topic. The MARPEX app is a student-oriented learning tool; hence, the proposed content has been verified and accepted by experts at every point of development. The experts involved are classified as content expert (Science Teacher) to review the content and design; and functionality experts (lecturer from relevant background) to test the suitability of the process of interaction of the MARPEX app.

The MARPEX app can only function with an AR marker. Figure 2 illustrates the AR marker used to access the virtual apparatus. Once the marker recognized, the MARPEX app displays the overlapping virtual apparatus (animated 3D model) on the mobile device screen. Photoshop software was used to customize the AR marker. A device database was created using the online database of Vuforia, and a new target was identified, and a unique name was given. For the MARPEX app, the targets include an animated 3D model for the experimental section, while text and audio included in the quiz section. The target sets the dimension or size, and then the target image file is uploaded to the Vuforia database. The compatible AR marker saved as a JPEG or PNG image file format using Vuforia. The Blender was used for modeling the $3 \mathrm{D}$ apparatus and for incorporating animation. Unity3D software has been used to integrate the animated 3D content of the MARPEX app with the augmented reality SDK.

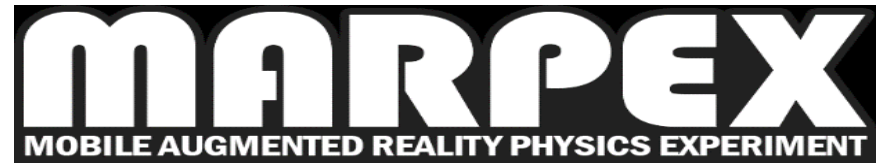

Fig. 2. Image-based markers for the MARPEX app

\subsection{Incorporation of MARPEX app on android device}

Vuforia SDK package imports into the development platform to create the augmented reality surface. Several features were determined for the MARPEX app, as discussed in the architecture of the MARPEX app. These features comprised of image targets, text targets, audio targets, animated 3D model targets, and the SDK project file for android development. The AR project marker file was downloaded from the Vuforia database after the custom image was uploaded as a target marker. A Unity Editor file was selected to match the authoring development of the Unity3D software. Subsequently, the unity editor format was set up with Vuforia SDK package, downloaded and import in Unity 3D for further development. The development of the MARPEX app requires the merging of Vuforia and Unity3D software. The C++ JavaScript used for further development of the application. The development of the MARPEX app that consists of 3D content making, animated the content, interaction and incorporation to the android device, test the functionality of Unity3D.

The main interface of the MARPEX app includes four main buttons consisting of three sets of experiments and a quiz. Further, two icons include; icon for navigation guide button for first time user as "i"(on the left side-bottom) and icon for the exit button as " $\mathrm{x}$ " (on the right side-top) were also included as shown in Figure 3. The designs of the icons designed and import in the Unity workspace. A transparent 
background was set in the Unity workspace, serving as the MARPEX app's background. Since the app requires a scene switch, an object named Manager created, and the script written in C++ JavaScript for the corresponding icon was attached to the object. Therefore, when an AR marker is scanned, the virtual apparatus that is attached to the AR marker appears on the android devices' screen.

MARPEX app intends to enhance students' motivation in science experiments through the use of the MAR platform. The MAR app is a tool that helps the students to improve the visualization and experiment skills in predicting the phenomenon that takes place and the possible outcome. All the MAR factors of the MARSELM conceptual model were encrypted in the design and development of the MARPEX app. The app comprises of virtual apparatus in the form of an animated 3D model, which were implied into the AR markers so that the students can view the enhanced contents when positioning the AR marker in front of their android device. Figure 3 shows the wireframe for the primary interfaces of the MARPEX app. The interfaces and steps in operating the experiment's animated 3D apparatus for each experiment have also been highlighted. Each experiment consists of several hypotheses where students can manipulate the manipulative variables to test their hypothesis for the respective experiment. In the meantime, the MARPEX app provides the user with enhanced visualization experience with instant virtual experiment experience. Conversely, it is essential to show the relationships between the MAR factors of the conceptual model and the developed app. The following section provides some grasps about the MAR factors of the MARSELM conceptual model that have been embedded into the app.

\section{$5 \quad$ Mar Factors of the Marselm Conceptual Model}

\subsection{Enjoyment}

Enjoyment is the state or process of the fun of something and the act of owning and benefiting from it. Enjoyment is defined as the emotion of excitement about the experience [34]. The enjoyment factor is crucial in the development of the MARPEX app as students quickly lose concentration and get bored. It is essential to add something enjoyable and cheerful to improve the enthusiasm of students in a science experiment. Enjoyment is always seen as the result of the fulfillment or fulfillment of one's desires, but some researchers have stated that pleasure goes beyond enjoyment, commitment, satisfaction and emotional fulfillment due to physical movement and activity [35], [36], and [37]. The emotional excitement using MAR has addressed in previous studies so far [4] and [38], and the enjoyment of physical movement has been largely unnoticed. Moreover, enjoyment is a good feeling that can reduce tension and increase learning motivation [39]. Hence, the MARPEX app drives the enjoyment factor with some great features like instant and manipulative variables, pan and rotation $3 \mathrm{D}$ tools, and monitoring controls while observing the flow of $3 \mathrm{D}$ animation tools while scanning the AR markers. 


\subsection{Realism}

Realism is the quality or the fact that a person or thing is represented in the right way of life. Realism can be categorized as the proper appearance and function of reallife equipment [40]. Meanwhile, using rendering methods such as Photo mapping, additional objects can be created to have a realistic look. Previously, there was a lack of reviews that considered the importance of realism in the appearance and functionality of motivational learning. [41] pointed out that "models are the recommended medium when realism is essential to learning." The more 3D tools in the real world, the more students can connect to the experiment internally. Therefore, the realistic factor included in the development of the MARPEX app was in terms of color and size of 3D apparatus, dynamism, sound effects, and $360^{\circ}$ viewpoints.

\subsection{Usability}

Usability, or can be considered as "ease of use" and "usability" focuses on evaluating interactive systems [42] and [43]. ISO defines usability as "the extent to which a product can be used by a particular user to achieve a specific goal with effectiveness, efficiency, and satisfaction in the context of specific use. "Usability" refers to methods for improving ease of use during the design process. Usability is embedded in the design of the interaction study, where interactions between users and applications occur. This study outlines that interactions between users and applications should be user-friendly and useful. Unnecessary interactions can lead to a negative impression. The usability factor is encrypted in terms of usefulness and ease of use where students can easily share applications, the app can operate in offline mode, and can re-do the experiments indefinitely. The transition from one 3D tool to another is done quickly on the same screen. The unnecessary transition screen and onscreen information are avoided in order to give students the space to build their own learning experiences.

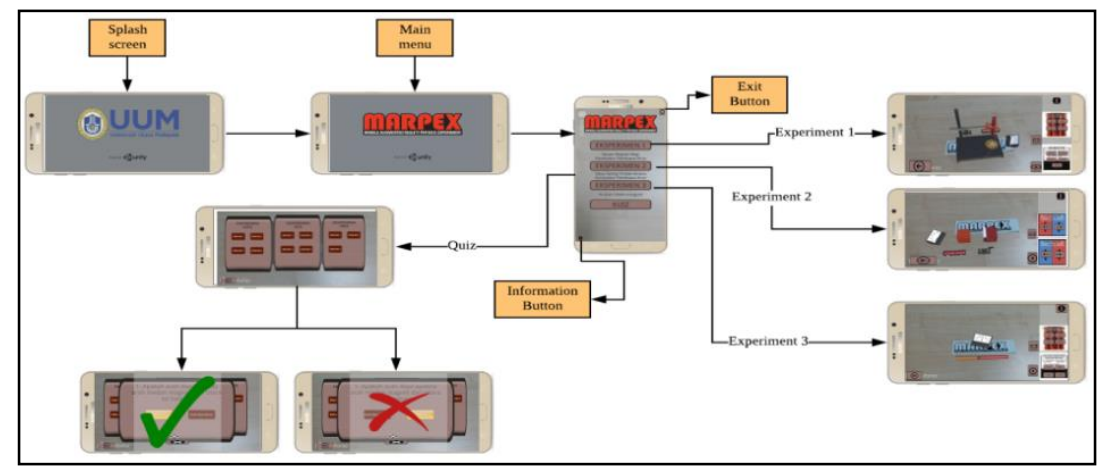

Fig. 3. Wireframe of the MARPEX app

The above discussion pertains to three key factors (enjoyment, realism and usability) of the MARSELM conceptual model introduced as of the MARPEX app 
after the development phase. [44] stated that an average human being acquired knowledge through visual senses (75 percent), hearing senses (13 percent) and other senses (12 percent). Thus, learning would be more productive and long-lasting if learning could trigger the senses, such as hearing, sight, touch [45] and [46]. The interplay of various features such as 3D modeling, animation, graphics and audio display in a technology-integrated learning environment is essential. The use of threedimensional (3D) models is vital because it provides an opportunity to visualize and experience learning from a variety of perspectives. 3D models may engage students, but static 3D models may not engage students continuously [22], [47] and [48]. Therefore, animation 3D models designed to attract attention and help to overcome the visualization problem. It also shows the ability of the MAR application in a science experiment to enhance student motivation.

\section{Conclusion and Future Work}

This paper has discussed and elaborated on the development of the MARPEX app in a science experiment to enhance students' motivation. The discussions on the technical requirements, the MARPEX app architecture, and the MARPEX app development continue to focus on the virtual content of the MARPEX app and the incorporation of MARPEX on an android device. Besides, there is a discussion on the three main MAR factors of the MARSELM conceptual model in the MARPEX app. Future work includes the evaluation of app users and experts. This paper is expected to guide future developments in the MAR app in a science experiment to enhance student motivation.

\section{$7 \quad$ Acknowledgement}

Our deepest gratitude goes to the Ministry of Education for supporting us by funding the Fundamental Research Grant Scheme (FRGS), and our utmost gratitude also goes to Universiti Utara Malaysia for other supports and facilities provided that have facilitated the research process along this year.

\section{$8 \quad$ References}

[1] G. J. Hwang and C. H. Chen, Influences of an inquiry-based ubiquitous gaming design on students' learning achievements, motivation, behavioral patterns, and tendency towards critical thinking and problem solving. British Journal of Educational Technology, 48(4), 950-971, 2017. https://doi.org/10.1111/bjet.12464

[2] H. S. Hsiao, C. S. Chang, C. Y. Lin and Y. Z. Wang, Weather observers: a manipulative augmented reality system for weather simulations at home, in the classroom, and at a museum. Interactive Learning Environments, 24(1), 205-223, 2016. https://doi.org/10. $\underline{1080 / 10494820.2013 .834829}$ 
[3] C. H. Chen, Y. Y. Chou and C. Y. Huang, an augmented-reality-based concept map to support mobile learning for science. The Asia-Pacific Education Researcher, 25(4), 567578, 2016. https://doi.org/10.1007/s40299-016-0284-3

[4] S. Kucuk, S. Kapakin and Y. Goktas, Learning anatomy via mobile augmented reality: effects on achievement and cognitive load. Anatomical sciences education, 9(5), 411-421, 2016. https://doi.org/10.1002/ase.1603

[5] W. K. Liou, K. K. Bhagat and C. Y. Chang, Beyond the flipped classroom: A highly interactive cloud-classroom (HIC) embedded into basic materials science courses. Journal of Science Education and Technology, 25(3), 460-473, 2016. https://doi.org/10.1007/ $\underline{\mathrm{s} 10956-016-9606-8}$

[6] M. Akcayir, G. Akcayir, H. M. Pektas and M. A. Ocak, Augmented reality in science laboratories: The effects of augmented reality on university students' laboratory skills and attitudes toward science laboratories. Computers in Human Behavior, 57, 334-342, 2016. https://doi.org/10.1016/j.chb.2015.12.054

[7] L. M. M. S. Al Qassem, H. Al Hawai, S. Al Shehhi, M. J. Zemerly and J. W. Ng, AIREDUTECH: Augmented immersive reality (AIR) technology for high school chemistry education. In Global Engineering Education Conference (EDUCON), 842-847, 2016. https://doi.org/10.1109/educon.2016.7474650

[8] M. B. Ibanez, A.Di-Serio, D. Villaran-Molina and C. Delgado-Kloos, Support for augmented reality simulation systems: the effects of scaffolding on learning outcomes and behaviour patterns. IEEE Transactions on Learning Technologies, 9(1), 46-56, 2016. https://doi.org/10.1109/tlt.2015.2445761

[9] S. S. Jamali, M. F. Shiratuddin, K. W. Wong and C. L. Oskam, Utilising mobileaugmented reality for learning human anatomy. Procedia-Social and Behavioral Sciences, 197, 659-668, 2015. https://doi.org/10.1016/j.sbspro.2015.07.054

[10] F. Ke and Y. C. Hsu, Mobile augmented-reality artifact creation as a component of mobile computer-supported collaborative learning. The Internet and Higher Education, 26, 33-41, 2015. https://doi.org/10.1016/j.iheduc.2015.04.003

[11] W. Tarng, K. L. Ou, C. S. Yu, F. L. Liou and H. H. Liou, Development of a virtual butterfly ecological system based on augmented reality and mobile learning technologies. Virtual Reality, 19(3-4), 253-266, 2015. https://doi.org/10.1007/s10055-015-0265-5

[12] H. T. Zimmerman, S. M. Land, M. R. Mohney, G. W. Choi, C. Maggiore, S. H., Kim. and J. Dudek, using augmented reality to support observations about trees during summer camp. In Proceedings of the 14th international conference on interaction design and children, 395-398, 2015. https://doi.org/10.1145/2771839.2771925

[13] J. Zhang, Y. T. Sung, H. T. Hou and K. E. Chang, The development and evaluation of an augmented reality-based armillary sphere for astronomical observation instruction. Computers \& education, 73, 178-188, 2014. https://doi.org/10.1016/j.compedu.2014.01. $\underline{003}$

[14] T. H. C. Chiang, S. J. Yang and G. J. Hwang, an augmented reality-based mobile learning system to improve students' learning achievements and motivations in natural science inquiry activities. Educational Technology \& Society, 17(4), 352-365, 2014.

[15] K.Tian, M. Endo, M. Urata, K. Mouri and T. Yasuda, Development of a Multi-viewpoint AR-Based Mobile Learning System for Supporting Lunar Observation. In International Conference on Signal-Image Technology \& Internet-Based Systems, 1034-1041, 2013. https://doi.org/10.1109/sitis.2013.167

[16] M. B. Ibáñez, Á. Di Serio, D. Villarán and C. D. Kloos, experimenting with electromagnetism using augmented reality: Impact on flow student experience and 
educational effectiveness. Computers \& Education, 71, 1-13, 2014. https://doi.org/10. 1016/j.compedu.2013.09.004

[17] J. Camba, M. Contero and G. Salvador-Herranz, Desktop vs mobile: A comparative study of augmented reality systems for engineering visualizations in education. In Frontiers in Education Conference FIE, IEEE, 1-8, 2014. https://doi.org/10.1109/fie.2014. $\underline{7044138}$

[18] H. Y. Wang, H. B. L. Duh, N. Li, T. J. Lin and C. C. Tsai, an investigation of university students' collaborative inquiry learning behaviors in an augmented reality simulation and a traditional simulation. Journal of Science Education and Technology, 23(5), 682-691, 2014. https://doi.org/10.1007/s10956-014-9494-8

[19] M. Kasahara, K. Takano, K. F. Li, A personalized learning system with an AR augmented reality browser for ecosystem fieldwork. In International Conference on Advanced Information Networking and Applications, 89-97, 2014. https://doi.org/10. 1109/aina.2014.16

[20] T. J. Lin, H. B. L. Duh, N. Li, H. Y. Wang and C. C. Tsai, An investigation of learners' collaborative knowledge construction performances and behavior patterns in an augmented reality simulation system. Computers \& Education, 68, 314-321, 2013. https://doi.org/10.1016/j.compedu.2013.05.011

[21] N. Li, Y. X. Gu, L. Chang and H. B. L. Duh, Influences of AR-supported simulation on learning effectiveness in face-to-face collaborative learning for physics. In Advanced Learning Technologies (ICALT), 320-322, 2011. https://doi.org/10.1109/icalt.2011. $\underline{100}$

[22] S. Oh and W. Woo, ARGarden: Augmented edutainment system with a learning companion. In Transactions on edutainment I, 40-50, Springer, Berlin, Heidelberg, 2008. https://doi.org/10.1007/978-3-540-69744-2 4

[23] L. A. Annetta and M. Shapiro, Augmented Reality Applications for Teaching Chemistry across the K-20 Curriculum. In Technology Integration in Chemistry Education and Research (TICER), 23-30, American Chemical Society, 2019. https://doi.org/10.1021/ bk-2019-1318.ch002

[24] B. L. Nielsen and H. Brandt, Augmented reality in lower secondary science teaching: Teachers and students as producers. In ESERA, 2019.

[25] D. D. McMahon, D. F. Cihak, R. E. Wright and S. M. Bell, Augmented reality for teaching science vocabulary to postsecondary education students with intellectual disabilities and autism. Journal of Research on Technology in Education, 48(1), 38-56, 2016. https://doi.org/10.1080/15391523.2015.1103149

[26] S. Sotiriou, S. Anastopoulou, S. Rosenfeld, O. Aharoni, A. Hofstein, F. Bogner and K. Hoeksema, Visualizing the invisible: The CONNECT Approach for teaching science. In Sixth International Conference on Advanced Learning Technologies, 1084-1086, IEEE, 2006. https://doi.org/10.1109/icalt.2006.1652636

[27] L. Kerawalla, R. Luckin, S. Seljeflot and A. Woolard, Making it real: exploring the potential of augmented reality for teaching primary school science. Virtual reality, 10 (34), 163-174, 2006. https://doi.org/10.1007/s10055-006-0036-4

[28] K. H. Cheng and C. C. Tsai, Affordances of augmented reality in science learning: Suggestions for future research. Journal of science education and technology, 22(4), 449462, 2013. https://doi.org/10.1007/s10956-012-9405-9

[29] S. S. Jamali, An investigation of mobile augmented reality-based learning features in cognitive and affective environments, Doctoral dissertation, Murdoch University, 2017.

[30] S. Siltanen, Theory and applications of marker-based augmented reality, Licentiate thesis, 2012. 
[31] J. A. A. Bakar, V. Gopalan, A. N. Zulkifli and A. Alwi, Design and development of mobile augmented reality for physics experiment. In International Conference on User Science and Engineering, 47-58, Springer, Singapore, 2018. https://doi.org/10.1007/978-981-13$1628-9 \_5$

[32] Vuforia Developer Portal. Retrieved from https://developer.vuforia.com, September 17, 2017.

[33] Unity-Download Archive. Retrieved from https://unity3d.com/get-unity/download/archive, September 17, 2017.

[34] R. Warner, Enjoyment. The Philosophical Review, 89(4), 507-526, DOI:10.2307/2184734, 1980.

[35] R. K. Dishman, R. W. Motl, R. Saunders, G. Felton, D. S. Ward, M. Dowda and R. R. Pate, Enjoyment mediates effects of a school-based physical-activity intervention. Medicine and science in sports and exercise, 37(3), 478-487, 2005. https://doi.org/10.1249/01.mss.0000155391.62733.a7

[36] S. T. Borra, N. E. Schwartz, C. G. Spain and M. M. Natchipolsky, Food, physical activity, and fun: Inspiring America's kids to more healthful lifestyles. Journal of the Academy of Nutrition and Dietetics, 95(7), 816, 1995. https://doi.org/10.1016/s0002-8223(95)00228-6

[37] S. Harter, Manual for the self-perception profile for children: (revision of the perceived competence scale for children). University of Denver, 1985.

[38] U. C. Pendit, S. B. Zaibon and J. A. A. Bakar, Conceptual model of mobile augmented reality for cultural heritage site towards enjoyable informal learning aspect. Jurnal Teknologi, 77(29), 123-129, 2015. https://doi.org/10.11113/jt.v77.6847

[39] H. Van der Heijden, User acceptance of hedonic information systems. MIS quarterly, 695704, 2004. https://doi.org/10.2307/25148660

[40] C. Chae and K. Ko, Introduction of physics simulation in augmented reality. In 2008 international symposium on ubiquitous virtual reality, 37-40, IEEE, 2008. https://doi.org/10.1109/isuvr.2008.17

[41] R. Heinich, M. Molenda, J. D. Russell and S. E. Smaldino, Instructional media and technologies for learning, Prentice-Hall, Inc., 1999.

[42] B. Shneiderman, Designing the user interface: Strategies for effective human-computer interaction. Addison Wesley Longman, USA, 1998.

[43] B. Shneiderman, Developing the User Interface. Addison-Wesley, USA, 1992.

[44] D. Laird and P. R. Schleger, Approaches to training and development. Reading, MA, Perseus Books, 1985.

[45] L. Biel, Students with Sensory Processing Challenges: Classroom Strategies. In Optimizing Learning Outcomes, 90-110, Routledge, 2017.

[46] T. Ha, Y. Lee and W. Woo, Digilog book for temple bell tolling experience based on interactive augmented reality. Virtual Reality, 15(4), 295-309, 2011. https://doi.org/10.1007/s10055-010-0164-8

[47] V. Gopalan, J. A. A. Bakar, A. N. Zulkifli and A. Alwi, A Review of Augmented Multimedia Elements in Science Learning. Journal of Telecommunication, Electronic and Computer Engineering (JTEC), 10(1-10), 87-92, 2018.

[48] S. Smith, Five components of multimedia. Retrieved from http://smallbusiness.chron .com/5-components-multimedia- 28279.html,20September.2017,202017.

[49] N. F. Saidin, N. D. Abd Halim and N. Yahaya, Framework for Developing a Mobile Augmented Reality for Learning Chemical Bonds. International Journal of Interactive Mobile Technologies (iJIM), 13(07), 54-68, 2019. https://doi.org/10.3991/ijim. $\underline{\mathrm{v} 13 \mathrm{i} 07.10750}$ 
[50] P. Boonbrahm, C. Kaewrat, P. Pengkaew, S. Boonbrahm and V. Meni, Study of the hand anatomy using real hand and augmented reality. International Journal of Interactive Mobile Technologies (iJIM), 12(7), 181-190, 2018. https://doi.org/10.3991/ijim. v12i7.9645

[51] F. N. Rusli, A. N. Zulkifli, M. N. Saad and Y. M. Yussop, (A Study of Students' Motivation in Using the Mobile Arc Welding Learning App. International Journal of Interactive Mobile Technologies (iJIM), 13(10), 89-105, 2019. https://doi.org/10. 3991/ijim.v13i10.11305

[52] V. Gopalan, A. N. Zulkifli and J. A. A. Bakar, A study of students' motivation using the augmented reality science textbook. In AIP Conference Proceedings, 1761(1), 1-6, AIP Publishing LLC, 2016. https://doi.org/10.1063/1.4960880

\section{Authors}

Valarmathie Gopalan obtained her bachelor degree in Multimedia and Master degree in Multimedia studies from Universiti Utara Malaysia (UUM). Currently, she is a Ph.D. student at the School of Multimedia Technology and Communication, Universiti Utara Malaysia, Malaysia. She has won gold medal for Mobile Augmented Reality Physics Experiment (MARPEX) application at Innovative Research, Invention, and Application Exhibition (i-ria) 2019. Her research interest includes multimedia, augmented reality, and mobile application. Email: valar83gopalan@gmail.com

Juliana Aida Abu Bakar currently is an associate professor at the School of Creative Industry Management \& Performing Arts, Universiti Utara Malaysia. She has won gold medals for innovative products in numerous exhibitions at national and international level and successfully lead two projects funded by national grants for the past five years. She has published over 100 articles in the renowned journals and proceeding with an h-index 6 . Her research interests include virtual reality design, development and evaluation augmented reality; and virtual heritage. Email: lianana@uum.edu.my

Abdul Nasir Zulkifli is an Associate Professor at the School of Creative Industry Management \& Performing Arts, Universiti Utara Malaysia. He has been teaching in various fields including Mechanical Engineering, Manufacturing Engineering, CAD, CAM, Management of Technology, Multimedia, Virtual Reality and 3D Animation for the last 34 years. His research areas include Virtual Reality, Augmented Reality, and Mobile applications in training and education. Email: nasirzul@uum.edu.my

Article submitted 2020-06-26. Resubmitted 2020-07-26. Final acceptance 2020-07-26. Final version published as submitted by the authors . 